\title{
Sterilization of Biofilm in Foam Using a Single Cavitation Bubble
}

\author{
Petr Schovanec ${ }^{1, *}$, Darina Jasikova ${ }^{1}$, Michal Kotek ${ }^{1}$, Karel Havlicek ${ }^{2}$, Magda Nechanicka ${ }^{2}$, \\ Jakub Eichler ${ }^{3}$, Jiri $\mathrm{Cech}^{3}$, and Petra Subrtova ${ }^{2}$ \\ ${ }^{1}$ Department of Physical Measurement, Technical University of Liberec, Institute for Nanomaterials, \\ Advanced Technology and Innovation, Studentska 1402/2, Liberec 1, 461 17, Czech Republic \\ ${ }^{2}$ Department of Environmental Technology, Technical University of Liberec, Institute for Nanoma- \\ terials, Advanced Technology and Innovation, Studentska 1402/2, Liberec 1, 461 17, Czech Republic \\ ${ }^{3}$ Faculty of Mechatronics Informatics and Interdisciplinary Studies, Technical University of Liberec, \\ Studentska 1402/2, Liberec 1, 461 17, Czech Republic
}

\begin{abstract}
This article presents the sterilization of bacteria using cavitation bubbles. Cavitation generated by ultrasound creates a cavitation cloud. Therefore is more advantageous to generate the cavitation bubbles by laser-induced breakdown, because it is possible to generate individual bubbles for the purpose of study single impact and physical mechanism of acting. The cavitation bubble is generated by a Nd: YAG $532 \mathrm{~nm}$ laser beam, a short 10ns pulse. Here, we used optics to focus the laser beam and a high-speed camera to visualize characteristics the bubble. We used the method of long-distance microscopy and shadowgraph lightening for the visualization. We used the particle image velocimetry (PIV) method to determine the interaction of the bubble with the surrounding liquid and solid surface. The main goal of the research is to use cavitation to sterilize bacteria and biofilm in impact of single bubble collapse on living cells.
\end{abstract}

\section{Introduction}

Cavitation is mostly famous as a negative effect in turbo machinery. These effects of cavitation have a potential for use in medicine, industry, biology, etc. Cavitation is used, for example, in the engineering industry for technological purposes such as cleaning shape complex parts, descaling and other impurities from the surface of products $[1,2]$.

Physical definition of cavitation phenomena is response of the liquid to the rapid changes of pressure. There are many ways how to generate bubbles. Bubbles should be generated by rotating propeller or vibrating surface. Another option is local deposition of energy or electrical discharge. Here is utilized laser pulse, spark, acoustic field, oscillations or boiling. In nature as in the fast river or in the waterfall and in real applications cavitation bubbles usually create a cloud, nevertheless, bubbles are not

\footnotetext{
*Corresponding author: petr.schovanec@tul.cz
} 
separated. The bubbles create structures which act collectively, however the essential elements of these structures are the individual bubbles [3, 4].

The convenient method for generating a single cavitation bubble is Laser Induced Breakdown (LIB). The single cavitation bubble can be very precisely geometrically placed in the volume of the liquid and close to the sample. The LIB method makes it possible to generate a cavitation bubble using ultrashort pulses of millijoules energies [6-9]. This method is thermal decomposition based on the generation of natural plasma. This plasma is in the form of optical breakdown, if the pulse exposure is from microseconds to femtoseconds. During the LIB method occurs direct, multiphoton and cascade ionization. Once the single bubble is stable, and of certain volume, it is either over heated, or expose to force impact. The significant role plays light wavelength, pulse width, impurities of the medium and spot size during the breakdown. Kennedy [7] explained the whole mechanism of the ionization very well.

\section{Experimental}

The experimental part is based on previous research $[4,5]$, which contains of bubble interaction towards a solid surface. In this experiment, we use the cavitation phenomena to eliminate bacteria. The main potential is in the use in wastewater treatment plants. Previously, sterilization of bacteria research has focused of using cavitation for defoaming and sterilization of the environment [13].

\subsection{Laser Induced Breakdown}

Laser-induced breakdown is a method in which laser light in a liquid is focused using appropriately designed optics. The laser induced breakdown in aqueous media and its collateral effects are described in detail by Kennedy in [7]. In addition it is followed by Vogel's work [8] on energy distribution during the growth and collapse of laser induced bubble. The authors investigated the influence of the laser pulse duration and input laser energy on the bubble dynamics and the shock waves emission.

\subsection{PIV setup}

The experiment contains two lasers, a camera and a function generator. One laser serves to generate plasma and cavitation bubbles in the liquid by means of the laser-induced breakdown the method. The second laser is used to illuminate a cuvette for camera to take photos. The function generator and time box serves time synchronization between the lasers and the camera. In this work we used high power laser with a short pulse $(t=10 \mathrm{~ns})$ and wavelength $(\lambda=532 \mathrm{~nm})$. A 10ns short laser pulse was generated using a Q-switched Nd: YAG Quantel EverGreen PIV pulse laser. This laser worked with one cavity to generate a single shot. Figure 1 presents schematic connection of experiment. 


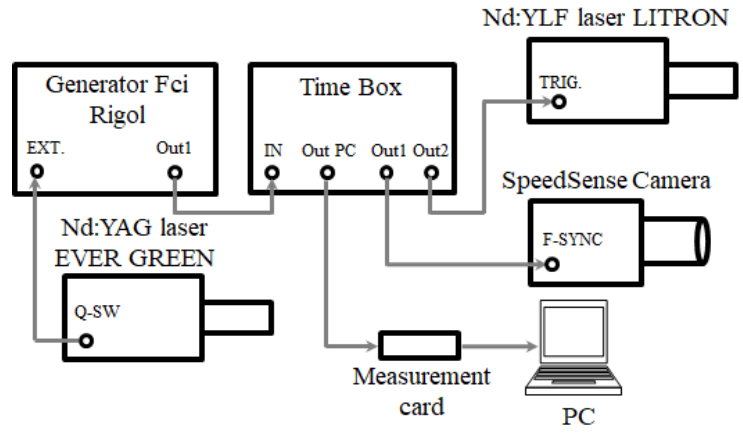

Fig. 1. Schematic connection of function generator, lasers and camera.

\subsection{Visualization setup}

We used shadowgraphy setup and method PIV for the bubble visualization. The camera exposure time was to $1 \mu \mathrm{s}$. The camera was mounted with optical lens system. The lens was fitted with edge pass and long pass filter cut-on wavelength $550 \mathrm{~nm}$ low pass optical filter to reduce the backward laser flashes to the camera and also to eliminate the flash generated while plasmatic breakdown. The beginning point of the synchronization was the plasma spot accompanied by the flash. The experimental setup is seen in Figure 2. The outlet diameter of the laser beam was $5 \mathrm{~mm}$ with Gaussian characteristics of the intensity. This arrangement was followed by a concave lens $f=30 \mathrm{~mm}$ and two plano-convex lenses $f=75 \mathrm{~mm}$ and $f=50 \mathrm{~mm}$ of $1 \mathrm{inch}$ diameter. The focused laser beam created the laser point - probe (diameter $<0.1 \mathrm{~mm}$ ).

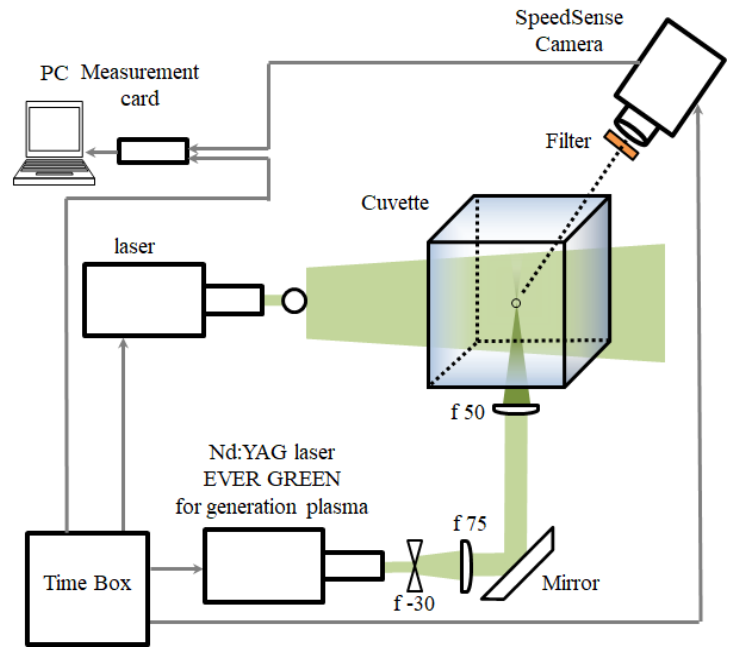

Fig. 2. The experimental setup.

\subsection{Bacterias}

A pure bacterial strain of Escherichia coli (EC; strain No.: CCM7395) and a foam from a wastewater treatment plant containing a mixture of fibrous bacteria (Microthrix, Gordonia, Skermania) were used to test the effect of cavitation on living systems. EC has been used for its general occurrence in the environment (parts of the human body, food, 
etc.), and its elimination is often required. Fibrous bacteria in the foam are highly resistant microorganisms, where their removal at wastewater treatment plants is necessary. Monitoring the influence of various parameters (settings) of generated cavitation bubbles on these bacteria gives an idea of their viability under given conditions in a certain environment [10-12].

The foam composed of bacteria and their resistant interconnected structures easily creates a clearly defined transition with the aqueous environment. This complex structure behaves similarly to a partially solid surface. In view of this fact, can be expected the behavior of cavitation bubbles as an impact towards a solid surface. However, this surface is not placed in a horizontal plane, but often inversely or vertically. Therefore, we can neglect the effect of the cavitation bubble due to the force of gravity. We focused on the behavior of the cavitation bubble with respect to the vertical location of the surface, both in terms of visualization and the study of the behavior of the liquid, which is locally accelerated by cavitation and exerts a force on a larger circle of cells than just the jet caused by bubble collapse.

\section{Result}

There is the plasma generated in the spot due the concentration of the laser energy and the plasma is visible due the emission. The pressure in liquid increase to $10^{3} \mathrm{bar}$, and temperature to $10^{3} \mathrm{~K}$ in the spot volume. This leads to plasma expansion at supersonic velocities, producing an acoustic shock wave followed by cavitation bubble effect. It is important to mark for the Figure 3 that the laser light comes from the bottom side in the pictures.

Figure 3 presents a shadowgraphy visualization series of images which has an exposure time of $10 \mathrm{~ns}$. The bubbles has been generated with a laser energy of $4 \mathrm{~mJ}$. The first frames in the time of $0 \mu \mathrm{s}$ show the position of the optical breakdown. The frame in the time of $60 \mu \mathrm{s}$ show the enlargement of the cavitation bubble in her maximum of diameter $1.4 \mathrm{~mm}$. The frame in the time of $120 \mu \mathrm{s}-255 \mu$ s show the collapse of the cavitation bubble towards a solid surface located on the right side in the images. The images was captured at a frequency of $68 \mathrm{kHz}$. A representative time-lapse of the bubble development and duration of individual phases is presented in Table 1.

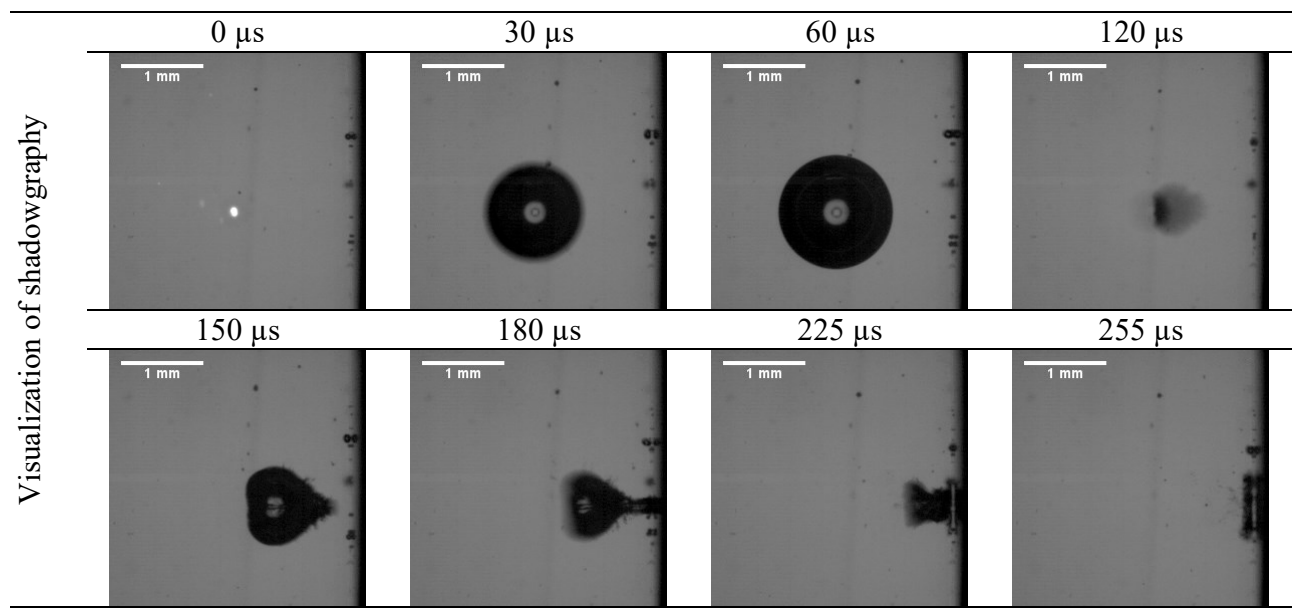

Fig. 3. Visualization of the plasma spot and the cavitation bubble. 
Table 1. Duration of expansion, collapse, and the impacting phase of the cavitation bubbles.

\begin{tabular}{|c|c|c|c|}
\hline $\begin{array}{c}\text { Spot Laser Energy } \\
(\mathrm{SLE})\end{array}$ & $\begin{array}{c}\text { Expansion Phase } \\
{[\mu \mathrm{sec}]}\end{array}$ & $\begin{array}{c}\text { Collapse Phase } \\
{[\mu \mathrm{sec}]}\end{array}$ & $\begin{array}{c}\text { Impacting / Jet } \\
\text { Phase }[\mu \mathrm{sec}]\end{array}$ \\
\hline $4 \mathrm{~mJ}$ & 60 & 120 & 180 \\
\hline
\end{tabular}

Figure 4 shows the calculation of the normalized velocity of the vector field and the vector field during the collapse of the cavitation bubble. The aim of the PIV method was to determine the interaction of the cavitation bubble with the surrounding liquid. It was captured by the PIV method the direction of the flow and the growth of the cavitation bubble and its collapse. Furthermore, the growth rate and collapse of the cavitation bubble were determined. The highest measured velocities achieve about $2-4 \mathrm{~mm} \cdot \mathrm{s}^{-1}$ during the growth and collapse of the bubble.

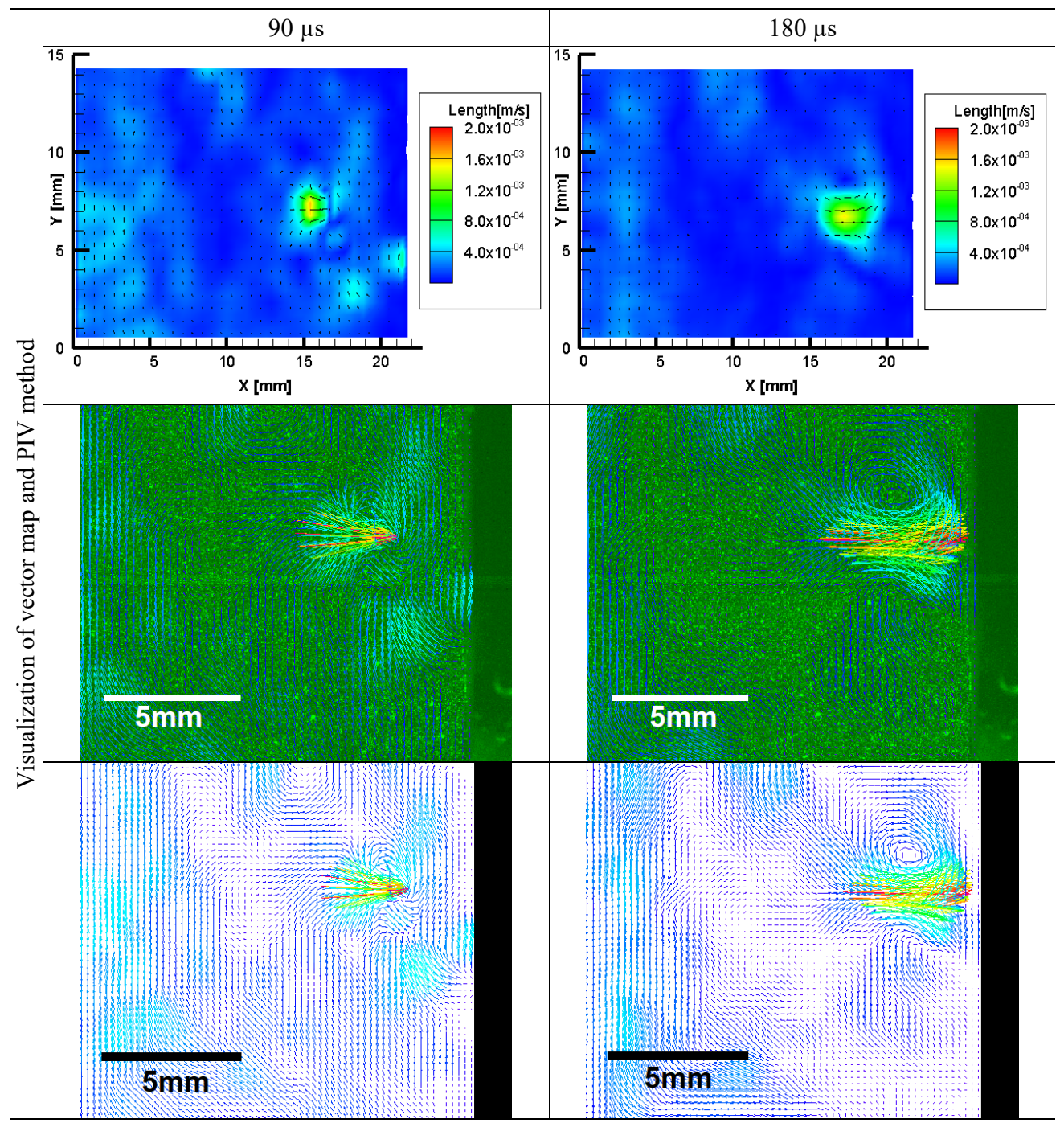

Fig. 4. Evolution in time of the cavitation bubble collapse, visualization of PIV method.

The EVER GREEN laser was used to generate a cavitation bubble, Litron laser for lighting and time box for synchronizing the SpeedSense high-speed camera with the Litron laser. 
With this connection, it was possible to work with a frequency in the range from unit $\mathrm{Hz}$ to $4 \mathrm{kHz}$, where the frequency could be further increased by reducing the exposure time of the camera, which was set from 100 to $300 \mu \mathrm{s}$. The solid surface was placed vertically at various distances from the bubble generating point, from 0 to $4 \mathrm{~mm}$.

During the study of the behavior of the cavitation bubble towards a solid surface, it was shown that the cavitation effect plays an important role in the elimination of bacteria. Images from the evaluation can be seen in Figure 5 and Figure 6.

The cavitation bubbles generated on the surface (sessile bubble) and in the distance is shown in Figure 5. The images show the intensity of the cavitation bubble energy impact during the collapse. The generation of sessile cavitation bubbles is preferred by most authors because a greater zone of impact is expected as the collapse of the bubble is fully projected onto the surface.

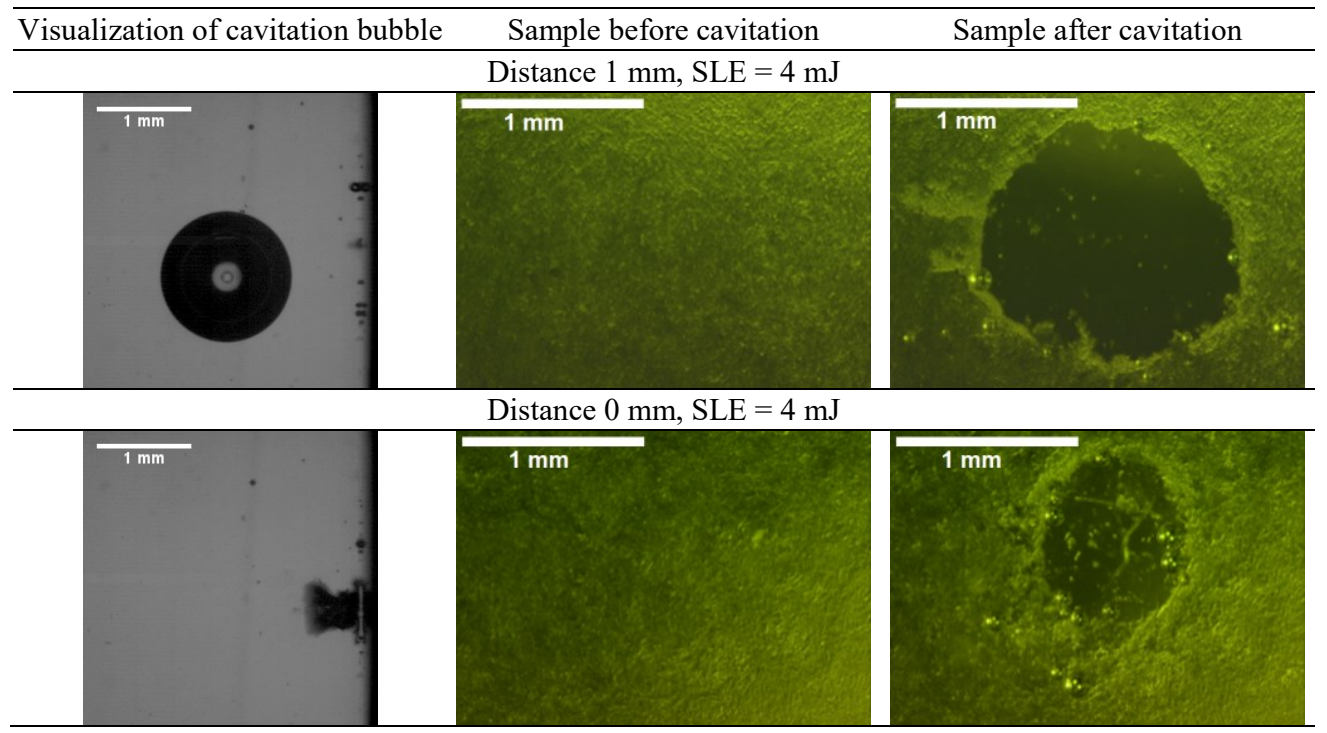

Fig. 5. Impact of the bubble cavitation.

Evaluation of live and dead bacteria was performed using confocal microscopy of biofilm. The green colour in the Figure 6 indicates live and red dead bacteria (microthrix, gordonia, skermania) contained in the foam.

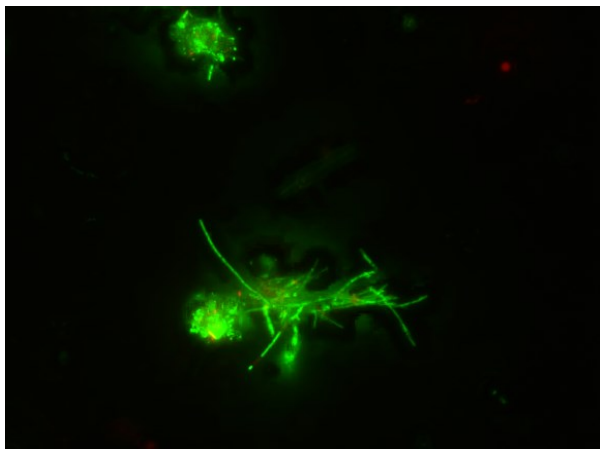

a)

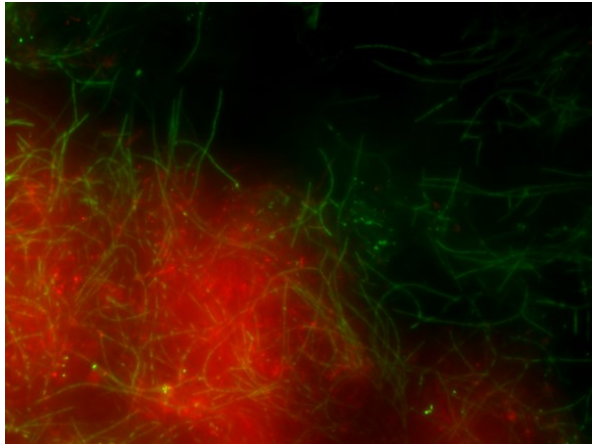

b)

Fig. 6. Live/dead bacteria; a) before cavitation, b) after cavitation. 


\section{Conclusion}

The visualization of cavitation bubbles is very important and useful tool in study of cavitation process. Here we use PIV method for calculation of the normalized velocity of the vector field where we determine the interaction of the cavitation bubble with surrounding and velocity of collapse cavitation bubble.

Cavitation was used for sterilization of biofilm in foam and to determine the most suitable and effective geometrical and input energy parameters of the system. In order to achieve this, we evaluated the setup based on the position of the cavitation bubble towards a solid surface at a distance of $0-4 \mathrm{~mm}$. The viability of the bacteria contained in foam (Microthrix, Gordonia, Skermania) depending on the properties of the cavitation bubble formation were studied.

During our research, it was shown that bacteria are eliminated by the cavitation effect. The cavitation effect plays an important role in the elimination of bacteria the effect was completely evident in Figure 5 and Figure 6.

This work was supported by the Ministry of Education, Youth and Sports of the Czech Republic and the European Union - European Structural and Investment Funds in the frames of Operational Programme Research, Development and Education - project Hybrid Materials for Hierarchical Structures (HyHi, Reg. No. CZ.02.1.01/0.0/0.0/16_019/0000843), and this work was partly supported by the Student Grant Competition of the Technical University of Liberec under the project No. SGS2020-3059.

\section{References}

1. D. Jasikova, P. Schovanec, M. Kotek, et al. Proceedings of SPIE, 10151, (2016)

2. D. Jasikova, P. Schovanec, et al. EPJ Web of Conferences, 143, (2017)

3. D. Jasikova, et al. EPJ Web of Conferences, 180, (2018)

4. P. Schovanec, W. Garen, S. Koch, et al. ACC JOURNAL 25, 58 (2019)

5. P. Schovanec, D. Jasikova, M. Kotek, V. Kopecky, EPJ Web of Conf. (to be published)

6. Ch. E. Brennen, Cavitation and bubble dynamics, (Cambridge University Press, 2015)

7. P. K. Kennedy, et al. Prog.QuantElectr. 21, 3, (1997)

8. Vogel, A., et al., Applied Physics B: Lasers and Optics 68, 271-280 (1999)

9. W. Lauterborn, et al. Archives of Acoustics 33, (2008)

10. K. Havlicek, L. Svobodova, et al. Materials \& Design, 194, (2020)

11. J. Nemecek, M. Nechanicka, et al. Chemosphere, 237 (2019)

12. K. Havlicek, M. Nechanicka, L. Svobodova, VTEI, 62, (2020)

13. J. B. Winterburn, P. J. Martin, Asia-Pacific Jrnl of Chem. Eng, 4, 184-190 (2009) 